\title{
Health psychology and consumers' perception of claims on food
}

\author{
G. Kavanagh ${ }^{1}$, F. Lalor ${ }^{1}$, J. Kennedy ${ }^{1}$, D. Minogue ${ }^{2}$ and P. G. Wall ${ }^{1}$ \\ ${ }^{1}$ School of Public Health and Population Science, University College Dublin, Dublin, Republic of Ireland and ${ }^{2}$ Psychology \\ Department, Trinity College, Dublin, Republic of Ireland
}

Over the past number of years there has been a substantial increase in the number and type of nutrition and health claims appearing on food labels and in 2006 a new European Regulation (EC 1924/2006) ${ }^{(1)}$ was introduced into law. The main aim of this new Regulation is to allow the 'average consumer' to make informed food choices. Thus, the consumer knowledge base and likely uptake of foods with health claims is an issue of pressing importance for food regulators and manufacturers.

The study consisted of 226 students. Participants were initially recruited by addressing a convenient sample of classes in the four major universities in Dublin and subsequently via the snowball method ${ }^{(2)}$. Validated scales were used to assess perceived dietary health ${ }^{(3)}$, perceived credibility of health claims and dietary knowledge ${ }^{(4)}$.

When asked about how credible they found a list of health claims, more participants were inclined to believe a health claim about 'probiotic cultures which aid healthy digestion' (60.0\%) than 'calcium to promote healthy bones and teeth' (57.8\%), 'plant stanols which help to reduce cholesterol' $(52.6 \%)$ or 'fibre which can help lower cholesterol' $(50.7 \%)$. Health claims were perceived as more credible when they were on yoghurt $(61.9 \%)$ than cheese $(59.3 \%)$, milk $(57.0 \%)$ or breakfast cereal $(55.9 \%)$. One-quarter $(25.5 \%)$ of participants reported that they had not bought any products with health claims in the last month. Of the participants who had purchased products with claims, those who purchased products to 'promote healthy bones and teeth' were more likely to purchase this product again (98.0\%). Pearson's product-moment correlation coefficient indicated a significant association between gender and purchase of products claiming to lower cholesterol $(r 0.15, n 226, P<0.05)$ but not for those promoting healthy digestion $(r-0.112, n 226, P=0.11)$ or healthy bones and teeth $(r 0.01, n 226, P=0.88)$. Males were more likely to purchase products with claims to lower cholesterol.

The present study shows that students are more inclined to believe health claims that have short-term health benefits rather than those with longer-term consequences. As shown previously ${ }^{(5)}$, the life-cycle position of individuals influences food choices. The present study has highlighted the way in which students perceive claims on food and further studies are needed to include a representative sample of 'average consumers' as indicated by the legislation.

1. European Commission (2006) Regulation (EC) No 1924/2006 of the European Parliament and of the Council of 20 December 2006 on nutrition and health claims made on foods. http://eur-lex.europa.eu/LexUriServ/LexUriServ.do?uri=OJ:L:2006:404:0009:0025:EN:PDF

2. Lopes CS, Rodrigues LC \& Sichieri R (1996) Int J Epidemiol 25, 1267-1270.

3. Hearty A, McCarthy SN, Kearney JM et al. (2007) Appetite, 48, 1-11.

4. Parmenter K, Waller J \& Wardle J (2000) Health Educ Res 15, 163-174.

5. Schafer RB \& Keith PM (1981) J Am Diet Assoc 78, 144-148. 